\title{
A crise financeira e a política econômica: poderia ter sido diferente?
}

\author{
João Ricardo M. G. Costa Filho*
}

\begin{abstract}
Resumo: Este artigo tem por objetivo analisar se a capacidade de resposta de política econômica foi fator relevante para minimizar a severidade da crise financeira de 2008, no primeiro ano do episódio. A hipótese é que países com mais espaço para políticas expansionistas registraram uma crise menos severa, tudo mais constante. Os resultados das regressões cross-country corroboram com a hipótese em relação à política monetária. No que diz respeito à política fiscal, possivelmente, mesmo países com bons resultados fiscais podem ter limitações a estímulos keynesianos em função da tolerância ao seu nível de endividamento. Entretanto, a interação entre o resultado do governo central e a dívida bruta está em linha com a hipótese da pesquisa, uma vez que uma melhor gestão tanto do fluxo fiscal, quanto do estoque da dívida no ano anterior ao evento mostrou-se relevante. A adição da variável de investment grade às especificações ressaltou uma crise mais severa nas economias desenvolvidas.
\end{abstract}

Palavras-Chave: Crise Financeira. Política Monetária. Política Fiscal.

\section{The financial crisis and the economic policy: could it have been different?}

Abstract: The aim of this paper is to analyse whether the economic policy response capability was a relevant factor for minimizing the 2008 financial crisis severity within its first year. The research hypothesis is that countries with a larger space for expansionary policies have registered a less severe crisis, holding everything else constant. The results from cross-country regressions corroborate with the hypothesis for the monetary policy. In relation to the fiscal policy, the sign of the parameters was the opposite of what was expected, signaling that, even countries with good fiscal results can experience limitations to Keynesian stimulus due to debt intolerances. However, the interaction between central govern result and gross debt confirms the research hypothesis, whereas a better management of the fiscal flow and debt stock simultaneously seems to be relevant. Adding an investment grade variable to the specifications highlighted that the crisis was more severe within the developed economies.

Keywords: Financial Crisis. Monetary Policy. Fiscal Policy.

Classificação JEL: C21, E52, E62, E63 e F30.

\section{Introdução}

Crises e episódios recessivos são recorrentes na economia internacional. Alguns exemplos, como a Crise de 1929, o choque do petróleo em 1973, a bolha da Nasdaq em 2000 ou o colapso no mercado norte-americano de hipotecas de alto risco em 2008, geraram um grande campo de pesquisa. Embora uma recessão típica dure cerca de quatro trimestres (resultando em uma perda acumulada de três pontos percentuais do PIB em relação ao pico

\footnotetext{
* Professor do Mestrado Profissional em Economia da EESP-FGV e Professor da Faculdade de Economia da FAAP. E-mail: joao.costa@fgv.br.
} 
anterior ao declínio, em média), ao associar a recessão a um episódio de contração no mercado de crédito o impacto verificado é maior que os eventos isolados (CLAESSENS, KOSE e TERRONES, 2008). As quedas no PIB decorrentes de crises de liquidez duram, em média, seis trimestres. O comportamento pró-cíclico do mercado de crédito e a sua influência na demanda agregada através do canal do consumo, ou por meio dos investimentos, explica porque eventos recessivos são amplificados quando ocorre uma crise de liquidez.

Em 2008, a combinação dos fatores acima descritos gerou a maior crise financeira após a Grande Depressão, afetando diversas economias ao redor do mundo. Passado o período de prosperidade econômica conhecido como a "Grande Moderação", a crise financeira que atingiu o mundo emergiu de uma correção nos preços dos imóveis no mercado norteamericano e se dissipou através dos mais complexos instrumentos derivativos, instaurando uma crise de confiança e de liquidez, trazendo sérias consequências para a economia real internacional.

A frequência de crises financeiras tem aumentado ${ }^{1}$ e os seus impactos por vezes são assimétricos. Os dados sobre a crise de 2008 mostram que a severidade difere entre países. Por quê? Talvez diferentes respostas de política econômica estejam por trás dessa dinâmica. Nesse contexto, este artigo pretende responder se essa capacidade de resposta minimizou a severidade da crise financeira de 2008 no seu primeiro ano. A hipótese é que países com taxas de juros mais altas - e, portanto, com maior espaço para expansões monetárias - e com melhores resultados fiscais, dispunham de uma maior capacidade de resposta, incorrendo em uma crise menos severa.

$\mathrm{O}$ artigo está dividio da seguinte forma. No capítulo a seguir, discutem-se algumas caracterísitcas da crise financeira de 2008 e sua relação com a literatura sobre crises e episódios recessivos. Partindo do ambiente macroeconômico de excesso de liquidez, passando pela dinâmica do mercado de imóveis norte-americano, até a transmissão por meio dos balanços dos bancos que resultou na contração do mercado de crédito, êxodo de capital e em problemas na economia real.

Feito o embasamento teórico, a hipótese de que países com maior espaço para a política econômica tiveram uma crise financeira menos severa é testada no terceiro capítulo, através de regressões cross-country, com estimadores de Mínimos Quadrados Ordinários. Como medida de severidade da crise foi utilizada a variação real do produto de 2009 frente a

\footnotetext{
${ }^{1}$ Eichegreen (2001) aponta para uma maior ocorrência de crises financeiras desde os anos 90. O autor ressalta que esse fator pode ser decorrente de maior contágio entre economias. Conforme Bordo and Murshid (2000), no entanto, a evidência é fraca.
} 
2008. Definiu-se como capacidade da política monetária o nível da taxa de juros em 2007. Para a política fiscal foi utilizado o resultado do governo central em 2007. Foi adicionada também uma variável de interação para incorporar a capacidade de expansão fiscal medida tanto pelo fluxo (resultado do governo central) quanto pelo estoque (dívida bruta em relação ao produto).

Os resultados das estimações corroboraram com a hipótese apresentada em relação à política monetária, na qual maiores taxas de juros resultariam em uma maior capacidade para estímulos expansionistas e, portanto, uma crise menos severa. Na política fiscal, mesmo países com uma boa situação fiscal podem ter espaço limitado para estímulos keynesianos. Contudo, a interação entre o resultado do governo central e o endividamento está em linha com a hipótese da pesquisa, uma vez que uma melhor gestão tanto do fluxo fiscal, quanto do estoque da dívida no ano anterior ao evento mostrou-se relevante.

Ao identificar o fenômeno do Global Savings Glut como importante para caracterizar a crise estudada, foi inserida nas regressões uma dummy para países considerados investment grade, sob a premissa de que países com esse tipo de classificação no final de 2007 teriam recebido um maior fluxo de capital e, no momento em que a crise instaurou um ambiente de aversão ao risco, o êxodo do capital pode ter feito com que esses países tivessem uma crise mais severa, o que fora comprovado nas estimações. A inclusão dessa variável deu maior poder explicativo às regressões (embora o objetivo do trabalho não seja a análise dos determinantes da crise financeira) e ressaltou que no seu primeiro ano foi mais severa nas economias desenvolvidas, o que faz sentido já que foi de onde emegiu.

\section{A Grande Recessão}

Como ressaltado na introdução, crises financeiras não só são recorrentes, como a sua frequência tem aumentado. A crise de 2008, contudo, ficará marcada como um dos maiores acontecimentos na história econômica. Além de ser a maior crise financeira global desde a Grande Depressão ${ }^{2}$, o desenrolar dos acontecimentos foi notável por inúmeras razões, incluindo sua severidade e velocidade ${ }^{3}$. Após um período de crescimento conhecido como a "Grande Moderação", o colapso no mercado de hipotecas de alto risco dos Estados Unidos desencadeou diversos acontecimentos que impactaram não só o mercado financeiro, mas também o lado real da economia. Eventos desestabilizadores de grande porte produzem

\footnotetext{
${ }^{2}$ Claessens, Kose e Terrones, (2008).

${ }^{3}$ Rose e Spiegel (2009).
} 
mudanças no desempenho de algumas variáveis macroeconômicas importantes no longo prazo (REINHART e REINHART, 2010).

No episódio, a idéia de alterações nos fundamentos macroeconômicos que antecederam a crise pode ser exemplificada pela a hipótese do Global Savings Glut, que entende o déficit em transações correntes norte-americano como resultado de uma maior poupança de agentes de outras nações (especialmente asiáticas $)^{4}$. O conseqüente influxo de capital nos EUA teria gerado uma maior oferta de recursos, diminuindo as taxas de juros da economia; além da incorporação de um grande player global - China - que pressionou para baixo os preços e em função da sua política cambial (CALOMIRIS, 2008).

Assim, um ambiente de excessiva liquidez estava instaurado. A taxa de juros norteamericana se encontrava em níveis bem baixos com base em simulações a partir da "regra de Taylor" (ROSE e SPIEGEL, 2009). Isso ocorreu não apenas pelo grande influxo de capital facilitado pelos novos instrumentos de securitização, mas também pela política monetária expansionista (BRUNNERMEIER, 2009). Além das praticas adotadas nos EUA, Taylor (2009) argumenta que condução da política monetária em outras economias também injetou liquidez no mercado financeiro internacional. $\mathrm{O}$ autor defende que desvios das taxas de juros aos níveis determinados pela "regra de Taylor" em diversos países seriam responsáveis pela valorização dos preços dos imóveisem diversos países. A Espanha - que teve o maior boom no mercado imobiliário - apresentava o maior desvio da regra. O país com o menor desvio, a Austrália, obteve a menor variação de investimento imobiliário como percentual do PIB.

Algumas mudanças no mercado financeiro contribuíram para a crise. O tradicional sistema no qual os bancos mantinham em seus balanços os empréstimos concedidos até que fossem liquidados deu lugar a um novo modelo no qual os empréstimos após concedidos eram "empacotados" e negociados via securitização. Essa inovação financeira tinha por objetivo tornar o sistema bancário mais estável, por meio da transferência do risco, mas propiciou uma expansão no crédito sem precedentes, além de ter auxiliado a fomentar uma bolha nos preços dos imóveis.

Com recursos em excesso e com os resultados da inovação financeira, novas práticas foram adotadas, como a mitigação do risco através de instrumentos derivativos, que diminuiu o custo dos empréstimos e estimulou concessões arriscadas por parte dos bancos (e.g. o caso dos clientes NINJAs, "no income, no job or assets"). As hipotecas eram concedidas sob a

\footnotetext{
${ }^{4}$ Bernanke (2005).
} 
premissa de que os preços das casas só poderiam subir e os mutuários sempre poderiam refinanciar as dívidas (BRUNNERMEIER, 2009).

A combinação de crédito barato com baixos padrões na concessão dos financiamentos foram os fundamentos da crise no mercado de hipotecas dos EUA (BRUNNERMEIER, 2009). A começar pela bolha imobiliária. Ao analisar o índice do mercado imobiliário criado pela Standard \& Poor's, o Case-Shiller Composite-20, utilizando como base 100 o mês de janeiro de 2000, constatou-se que até julho de 2006 os imóveis obtiveram uma valorização de $106,5 \%$.

Nem todos os agentes estavam convencidos de que o movimento de alta não era justificado. Alguns especialistas argumentavam que o período de valorização dos preços dos imóveis não configurava uma bolha especulativa, e sim estava fundamentado no crescimento da produtividade norte-americana e na queda do risco acompanhado pela "Grande Moderação" na volatilidade macroeconômica (REINHART e ROGOFF, 2008). Contudo, a correção ocorreu rapidamente. Em novembro de 2008 as residências já tinham perdido um quarto do seu valor.

A literatura econômica mostra que, em geral, na década que antecede uma crise financeira, o volume de recursos frente ao produto acumula um crescimento de $38 \%$ no período. Após o choque a contração dos recursos historicamente é também de 38\% (REINHART e REINHART, 2010). Crises bancárias costumam ser precedidas por um período prolongado de crescimento do mercado de crédito e geralmente associadas a problemas nos balanços de empresas do setor privado, como descasamento de prazos entre ativos e passivos, ou risco cambial, que em última instância se transferem para o risco de crédito do sistema bancário (LAEVEN e VALENCIA, 2010).

O trigger da crise foi o aumento na inadimplência das hipotecas. O impacto do foi amplificado pelos instrumentos financeiros conhecidos como derivativos. Através do sistema de "empacotamento e distribuição" os bancos aumentaram a concessão de empréstimos em função da aparente redução de risco através das securitizações. Adicionalmente, as diferenças nas maturidades entre os ativos e passivos era gerenciada através dos derivativos (BRUNNERMEIER, 2009). Contudo, a utilização dessas estruturas complexas dificultou a avaliação da exposição das instituições e a dificuldade na identificação de quais instituições estavam com problemas foi fator importante durante a crise (TAYLOR, 2009).

É comum em recessões severas que tanto os preços dos imóveis, como o valor dos ativos financeiros se contraiam (diminuição de $55 \%$ e $60 \%$ em relação ao pico, 
respectivamente). Os preços dos ativos apresentam o maior grau de sincronização - este podendo ser entendido pela quantidade de países vivenciando o mesmo tipo de comportamento em seus respectivos mercados - refletindo a integração entre os mercados financeiros. (CLAESSENS, KOSE e TERRONES, 2008).

Além da contração no mercado imobiliário, o mercado de ações também sofreu uma forte correção. Entre outubro de 2007 e outubro de 2008 a queda no valor das empresas listadas nos EUA foi de oito trilhões de dólares (BRUNNERMEIER, 2009). Os efeitos no balanço patrimonial podem ter causado espirais de liquidez. Após a queda no valor dos ativos, o capital da instituição financeira diminui, mas o nível de empréstimos se mantém. Inicia-se então uma venda dos ativos em carteira para obtenção de capital, o que pressiona os preços dos ativos e piora a situação de financiamento do banco. Os pedidos de aumento de margem reforçam essa espiral de liquidez.

Outra forma de transmissão ocorreu através dos canais de empréstimo. A incerteza por parte dos bancos sobre o acesso ao mercado de capitais no futuro faz com que os mesmos mantenham um colchão de liquidez maior do que o normal, diminuindo a concessão de recursos. Pode-se atribuir esse movimento ao moral hazard no monitoramento dos empréstimos - a diminuição do monitoramento força o mercado a se adaptar e tornar-se mais restrito nas concessões de recursos - e às medidas preventivas a possíveis choques de mercado (BRUNNERMEIER, 2009). A concentração que surge quando os bancos são tomadores e fornecedores de recursos também pode ter amplificado os problemas que tiveram origem no mercado de hipotecas dos EUA.

As respostas de política econômica na Crise Financeira de 2008 contêm o mesmo tipo de ferramentas que foram utilizadas nas crises passadas. Os policymakers se utilizam de extensivos suportes à liquidez e garantias. Contudo, uma das diferenças está na velocidade de resposta e na implementação dos instrumentos utilizados (LAEVEN e VALENCIA, 2010). A política monetária foi significativamente expansionista ajustando rapidamente a taxa de juros de curto prazo através de uma ação coordenada entre diversos bancos centrais (CLAESSENS et al, 2009). O Federal Reserve (Banco Central dos EUA) adotou medidas como o Term Auction Facility (TAF), que possibilitava aos bancos a obtenção de recursos diretamente do Banco Central norte-americano, sem utilizar a taxa de redesconto, para reduzir os spreads do money market, além do corte na taxa de juros dos federal funds.

O Tesouro norte-americano contribuiu com pacotes fiscais como o Economic Stimulus Act, estímulo iniciado em fevereiro de 2008 por meio do envio de recursos para as famílias 
nos EUA com objetivo de impulsionar o consumo (TAYLOR, 2009). Uma política fiscal acomodativa foi importante na manutenção da demanda agregada e suportou as instituições financeiras indiretamente. Diversos gestores de política econômica optaram por políticas anticíclicas através de medidas fiscais. Ao suportar a demanda agregada, o estímulo fiscal ajuda a reduzir a inadimplência, diminuindo a necessidade de recapitalização dos bancos (CLAESSENS et al, 2009).

Embora o trigger seja de origem microeconômica, as consequências macroeconômicas tanto nos EUA, como em diversos países foram relevantes. A abertura financeira e comercial que vem se desenvolvendo desde o fim do regime de Bretton-Woods deixou as economias mais expostas às recessões nos seus parceiros comerciais e provedores de capital. As economias emergentes foram afetadas por choques externos através de dois canais.

O primeiro canal foi o da (rápida) redução das exportações - para os produtores de commodities queda nos termos de troca. O segundo foi a queda abrupta no influxo líquido de capital (BLANCHARD et al., 2010). Em meio a um ambiente de incerteza, a contração no mercado de crédito com os bancos estrangeiros restringiu os empréstimos e fez com que países dependentes de recursos de terceiros externos sofressem com a escassez de liquidez (LANE e MILESI-FERRETI, 2010).

A interdependência entre os países fez com que a crise financeira se espalhasse com mais intensidade do que os economistas pudessem ter antecipado (KAMIN e DEMARCO, 2010). O capital "repatriado" no processo de desalavancagem das instituições financeiras que intermediavam os investimentos internacionais auxiliou na "exportação" da crise (IMBS, 2009). Além do revés nas finanças internacionais, a economia real também auxiliou na propagação da crise. A mudança abrupta na tolerância às incertezas atingiu fortemente os países que incorriam em grandes déficits em conta corrente, grande passivo externo líquido ou grande passivo bruto com desbalanceamento nos prazos ou na denominação da dívida, principalmente após um período extenso de subavaliação de risco nos anos anteriores à crise. Diante do ambiente de incerteza e da restrição do crédito, a demanda por bens duráveis contraiu e o comércio internacional como um todo colapsou (LANE e MILESI-FERRETI, 2010).

\section{Análise Empírica}

A crise financeira foi um fenômeno global, mas a sua severidade diferiu entre os países. Uma possível explicação estaria nas restrições encontradas pelos policymakers. 
Embora tanto a política monetária quanto a política fiscal tendam a ser anticíclicas em episódios como os analisados (CLAESSENS, KOSE e TERRONES, 2008), podem existir limitações. Será que a capacidade de resposta da política macroeconômica foi fator relevante para minimizar os efeitos da crise financeira de 2008 no primeiro ano após o evento?

Para responder essa pergunta considera-se como severidade da crise a variação do PIB no ano de 2009 frente ao ano de 2008. Como os governos possuem diversos instrumentos para responder às adversidades macroeconômicas, tanto no que tange a política fiscal, quanto em relação às ferramentas de política monetária, uma aproximação para a análise das políticas fiscal e monetária é a avaliação do consumo do governo e do comportamento da taxa de juros de curto prazo, respectivamente (CLAESSENS, KOSE e TERRONES, 2008).

A hipótese da pesquisa baseia-se na idéia de que i) taxas de juros mais altas representam uma maior capacidade de resposta de política monetária porque quanto maior o nível pré-crise, maior é espaço para cortes na mesma e, portanto, o coeficiente da variável na regressão deveria ser positivo; ii) analogamente, governos que incorrem em melhores resultados nominais poderiam responder à crise através de estímulos fiscais mais facilmente, portanto, o coeficiente desta variável também deveria ser positivo. A equação abaixo representa o modelo estimado:

$$
Y_{i, 09 / 08}=\beta_{0}+\beta_{1} \cdot F_{i}+\beta_{2} \cdot M_{i}+\sum_{k} \phi^{k} \cdot C_{i}^{k}+\varepsilon_{i}
$$

Para

\section{$\mathrm{i}=1,2, \ldots, \mathrm{N} ; \mathrm{N}$ é o número de países da amostra}

onde $\mathrm{Y}_{\mathrm{i}, 09 / 08}$ representa a variação percentual do produto interno bruto de 2008 a 2009. As variáveis de interesse, $\mathrm{F}_{\mathrm{i}}$ e $\mathrm{M}_{\mathrm{i}}$, representam o resultado fiscal do governo central no ano de 2007 em proporção ao PIB e o nível da taxa de juros no final do ano de 2007, respectivamente; $\mathrm{C}_{\mathrm{i}}^{\mathrm{k}}$ é o vetor de variáveis de controle; $\varepsilon_{\mathrm{i}}$ é o termo de erro da regressão; $\beta_{0}, \beta_{1}, \beta_{2}$, representam o interceprto e os coeficientes das variáveis das variáveis de interesse. Finalmente, $\phi^{k}$ representa o coeficiente de cada uma das $\mathrm{k}$ variáveis de controle.

Foi utilizada a razão dívida bruta/PIB para controlar os efeitos do endividamento do país nos níveis de taxas de juros e no resultado do governo central. Utilizou-se também uma variável resultante da interação entre o resultado do governo central em 2007 e a razão dívida bruta/PIB no mesmo ano. Outra variável de controle foi a razão crédito privado/PIB, por ser 
uma medida de solvência que interfere tanto na política monetária, como na política fiscal em função do caráter pró-cíclico do mercado de crédito e da transmissão dos efeitos monetários pelo mesmo (KIYOTAKI e MOORE, 1997). Na mesma linha, a variação dessa razão foi utilizada como regressor, pois a velocidade do desenvolvimento do mercado de crédito pode impactar as ações de política econômica.

A volatilidade do PIB foi incorporada na regressão dado que a política econômica tende a suavizar os ciclos de negócio, uma maior volatilidade influenciaria no nível dos instrumentos de política econômica. O crescimento médio do PIB entre 1990 e 2007 foi incorporado como uma medida de tendência. Uma dummy para exportadores de petróleo foi incorporada para extrair os impactos da mudança dos preços do petróleo nas nações exportadoras do produto (LANE e MILESI-FERRETI, 2010).

Foram utilizadas as variáveis PIB per capita e crescimento do PIB per capita para controlar os efeitos do desenvolvimento econômico ${ }^{5}$. A idéia é que a gestão de política econômica difira em países mais ou menos em distintos estágios de desenvolvimento, impactando as taxas de juros e os gastos do governo. A variável abertura comercial capta a interação comercial da economia com o resto do mundo e a variável conta corrente/PIB incorpora uma possível vulnerabilidade externa a choques internacionais.

O controle pelo regime cambial captura os possíveis efeitos na política econômica em decorrência da escolha de um regime mais fixo ou mais flexível da taxa de câmbio ${ }^{6}$. Em regimes cambiais mais rígidos, espera-se uma maior propensão a crises em comparação a regimes com câmbio flutuante, principalmente em economias emergentes com um maior grau de integração internacional, pois estão mais vulneráveis à choques nos fluxos de capitais (BUBULA e OTKER-OBE, 2003).

\subsection{Amostra}

A amostra é composta por 183 países $^{7}$, limitada pela disponibilidade de dados nas bases utilizadas. Os dados referentes às taxas de juros, resultados do governo central, dívida bruta sobre o PIB e índice de preços ao consumidor (CPI - Consumer Price Index) foram

\footnotetext{
${ }^{5}$ Lane e Milesi-Ferreti (2010).

${ }^{6}$ Regime cambial de 2007 com base na metodologia proposta por Reinhart e Rogoff (2007).

7 Vide quadro 2 no apêndice.
} 
obtidos nos sites do Fundo Monetário Internacional (FMI) e do Banco Mundial ${ }^{8}$. Em média a variação do PIB de 2009 frente ao ano de 2008 foi de 0,03\%, com desvio-padrão de 5,14\%. O país com o maior crescimento foi Timor-Leste, crescendo 12,37\%. A Latvia apresentou a maior queda no PIB, $17,95 \%$. Os países de alta renda ${ }^{9}$ registraram uma crise mais severa em relação ao comportamento do PIB, com contração média de 3,08\%. Já os países de baixa renda tiveram crescimento econômico - em média de 3,77\%.

A taxa de juros nominal média em 2007 foi de 6,01\% ao ano, com desvio-padrão de 3,81\%. Países como o Sri Lanka, com taxa de juros de 30,88\% ao ano, e o Japão com $0,47 \%$ destacam-se nos extremos. Nos países de alta renda, a média foi de 4,59\% ao ano. Os países de baixa renda tiveram uma taxa média de 6,42\%. A taxa de juros real ex-post média foi de $0,77 \%$, com desvio-padrão de $3,86 \%^{10}$. O Chad apresentou a maior taxa de juros real, $14,53 \%$ ao ano, e a Ethiopia a menor (-11,20\% ao ano). Ao analisar somente os países com alta renda, a média é de $1,27 \%$. Para os países de baixa renda a média é de $0,70 \%$. Possivelmente, os países de baixa renda apresentaram menores taxas de juros reais em 2007 em função de maiores taxas de inflação.

Em relação ao resultado do governo em 2007 em proporção do PIB, a média da amostra foi de $3,3 \%$, com desvio-padrão de 26,28\%. O maior resultado foi do Timor-Leste apresentou um resultado fiscal equivalente a $316 \%$ do seu produto. No outro extremo, o Kiribate apresentou déficit de 11,20\% do PIB. Para os países de alta renda, a média ficou em 3,95\% do PIB. Os países de baixa renda registraram média de 1,49\% do PIB. No que tange o endividamento bruto, em média os países da amostra apresentaram um nível de dívida/PIB de $51 \%$, com desvio-padrão de 53\%. O país com o maior endividamento foi a Libéria, com $541 \%$ do PIB. A Guinea Equatorial foi o país da amostra com a menor relação dívida/PIB em 2007, de $1 \%$. Os países de alta renda registraram uma média de $45 \%$ de endividamento bruto sobre o PIB. Já os países de baixa renda apresentaram uma dívida média de $68 \%$ do PIB.

No que diz respeito ao crédito privado sobre o PIB, a média foi de 54\%, com desviopadrão de 50\%. A Islândia figura com a maior porcentagem de crédito em relação ao produto, $270 \%$. O Sudão tem a menor razão, próxima a zero. Nos países de alta renda a razão é de $108 \%$ em média. Para os países de baixa a renda, a média é de $17 \%$ do PIB. A crise de 2008 mostrou que além do acompanhamento do nível de crédito doméstico, a sua variação também

\footnotetext{
${ }^{8}$ A relação das fontes encontra-se no quadro 1. As demais variáveis foram obtidas na base de dados utilizada no artigo "The Cross-Country Incidence of the Global Crisis". O autor agradece a gentileza de Gian Maria MilesiFerretti.

${ }^{9}$ Classificação do Banco Mundial.

${ }^{10}$ Calculada com base na taxa de juros nominal, deflacionada pelo Consumer Price Index pelo autor.
} 
é importante. Uma grande expansão em um curto intervalo de tempo pode implicar na formação de bolhas no mercado de crédito. No que tange a variação do crédito privado sobre o PIB entre 2003 e 2007, o incremento médio foi de nove pontos percentuais, com desvio padrão de 20 pontos percentuais. O maior aumento ocorreu na Islândia, 153\%. No outro extremo está Singapura, com queda de $37 \%$. Nos países de alta renda, o aumento médio foi de $16 \%$, já nos países de baixa renda, apenas $3 \%$.

A volatilidade média do produto medida pelo desvio-padrão da variação do crescimento do PIB dos países da amostra é de 4,02 (com desvio padrão de 3,59). O país que apresentou maior variabilidade entre 1990 e 2007 foi a Guinea Equatorial, 35,53. O Zimbábue foi o país com menor volatilidade no período, 3,13. Países com alta renda apresentaram uma volatilidade do produto média $(3,55)$ menor do que países com baixa renda $(4,56)$. Em relação ao crescimento médio do PIB entre 1990 e 2007, os países da amostra obtiveram uma média de 4,19\% ao ano, com desvio-padrão de 2,77\%. O país com maior crescimento médio foi a Guinea Equatorial, com crescimento médio de 27,76\% ao ano. O Zimbábue apresentou o maior decréscimo médio no período, 1,98\% ao ano. Os países de alta renda apresentaram um crescimento médio de 4,28\% ao ano, superior ao crescimento médio dos países de baixa renda, de $3,67 \%$ ao ano.

O PIB per capita médio da amostra é de US\$ 11.939,44, com desvio padrão de US\$ 18.094,68. O país com o maior produto por habitante é Luxemburgo (US\$ 106.983,30). No outro extremo está o Burundi (US\$ 125,07). Nos países de alta renda a média é de US\$ 36.699,67. Nos países de baixa renda, a média é de US\$ 588,18. O crescimento médio do PIB per capita de 2003 a 2007 foi de 74\%, com desvio-padrão de 52\%. O país que registrou o maior aumento foi Azerbaijão - 335\%. O país com o menor crescimento foi o Japão - 3,4\%. Os países de alta renda tiveram um crescimento médio do PIB per capita de $61 \%$. Os países de baixa renda apresentaram crescimento médio de $63 \%$ - países de renda intermediária experimentaram crescimento de $87 \%$, em média.

O resultado da conta corrente em relação ao PIB médio dos países analisados é negativo em 1\%, com uma grande dispersão (desvio-padrão de 27\%). O país com maior superávit em proporção ao produto é o Timor-Leste com 296\%. Grenada foi o país que apresentou o maior déficit em relação ao PIB, $43 \%$ aproximadamente. Os países de alta renda apresentaram um resultado em conta corrente de $4 \%$ do PIB, em média. Já os países de baixa renda registraram um déficit médio em conta corrente de $1 \%$ do PIB, em linha com a idéia da exportação de poupança de países ricos para economias em desenvolvimento. 
Em relação à abertura comercial em média a soma de exportações e importações em relação ao PIB é de 99,33\%. Hong Kong possui o maior grau de abertura comercial (405\% do PIB). O Brasil figura com o menor grau (25,2\% do PIB). A média para os países de alta renda é de $120 \%$. Os países de baixa renda apresentam um grau de abertura comercial de $79,82 \%$ do PIB em média. A tabela 1 resume as estatísticas descritivas sobre as variáveis analisadas.

Tabela 1: Estatísticas Descritivas

\begin{tabular}{|c|c|c|c|c|c|c|}
\hline Variável & Observações & Média & Desvio-padrão & Mediana & Máximo & Mínimo \\
\hline$\Delta \mathrm{PIB}_{09 / 08}$ & 176 & 0.03 & 5.14 & 0.18 & 12.94 & -17.96 \\
\hline Taxa de Juros Nominal & 128 & 6.01 & 3.81 & 4.87 & 30.88 & 0.47 \\
\hline Taxa de Juros Real & 127 & 0.77 & 3.86 & 1.26 & 14.53 & -11.20 \\
\hline Resultado Fiscal & 176 & 3.30 & 26.28 & -0.22 & 315.99 & -15.90 \\
\hline Dívida/PIB & 163 & 0.51 & 0.53 & 0.39 & 5.41 & 0.01 \\
\hline Crédito Privado/PIB & 152 & 0.54 & 0.50 & 0.36 & 2.70 & 0.00 \\
\hline$\Delta$ Crédito Privado/PIB ${ }_{(2003-2007)}$ & 151 & 0.09 & 0.20 & 0.05 & 1.53 & -0.37 \\
\hline Volatilidade do PIB $_{(1990-2007)}$ & 176 & 4.03 & 3.59 & 3.13 & 35.53 & 0.18 \\
\hline Crescimento Médio do $\mathrm{PIB}_{(1990-2007)}$ & 176 & 4.20 & 2.78 & 3.82 & 27.76 & -1.98 \\
\hline PIB per capita & 176 & 11,792 & 18,040 & 3,810 & 106,983 & 125 \\
\hline Crescimento Médio do PIB per capita $(2003-2007)$ & 172 & 0.74 & 0.52 & 0.59 & 3.35 & 0.03 \\
\hline Conta Corrente/PIB & 177 & -0.01 & 0.27 & -0.04 & 2.96 & -0.43 \\
\hline Regime Cambial & 157 & 1.95 & 0.99 & 2.00 & 6.00 & 1.00 \\
\hline Abertura Comercial & 162 & 99.33 & 55.46 & 86.65 & 405.11 & 25.20 \\
\hline
\end{tabular}

Fonte: FMI e Banco Mundial; Elaboração do Autor.

\subsection{Resultados Econométricos}

O método escolhido para verificação das hipóteses apresentadas foi o dos mínimos quadrados ordinários (MQO) $)^{11}$ na mesma linha do trabalho desenvolvido por Lane e MilesiFerreti (2010). Duas especificações foram estimadas através do software Stata 9.0. As regressões (1) e (2) contêm a variação do PIB como variável endógena e diferem apenas na variável de política monetária - taxa de juros nominal e taxa de juros real, respectivamente.

Com base nos resultados das regressões a taxa de juros nominal apresentou coeficiente positivo, em linha com a hipótese apresentada de que uma maior capacidade de resposta de política monetária poderia minimizar os efeitos da crise financeira. Contudo, não foi estatisticamente significativo. A taxa de juros real apresentou coeficiente negativo, também não significativo estatisticamente.

\footnotetext{
${ }^{11}$ Estimações robustas à heterocedasticidade.
} 
A variável de política fiscal apresentou coeficiente estatisticamente significativo nas duas regressões, com significância de $1 \%$ em ambas. O sinal contraria a hipótese apresentada, de que um resultado positivo indicaria que maior espaço para estímulos fiscais resultaria em uma crise menos severa, tudo mais constante. Uma possível explicação para o resultado negativo seria a hipótese de que mesmo países com bons resultados fiscais tenham limites para expansões fiscais. Esses limites podem emergir em decorrência de diferentes níveis de tolerância ao endividamento dos países.

A variável de interação fiscal apresentou coeficiente positivo e significativo - a $5 \%$ e $1 \%$ nas regressões (1) e (2), respectivamente - em linha com a hipótese de trabalho de que uma melhor situação fiscal - combinando o fluxo com o estoque de dívida - minimizaria os efeitos da crise financeira, indicando uma maior capacidade de resposta para a política fiscal. Países com histórico de default e que registraram altas taxas de inflação possuem uma maior intolerância por parte dos investidores, do que países com uma dinâmica de preços e de pagamento de dívidas mais favorável (REINHART, ROGOFF e SAVASTANO, 2003).

A razão dívida/PIB apresentou coeficiente negativo na regressão com a taxa de juros nominal, mas não foi estatisticamente significativo. O sinal do coeficiente aponta para a dificuldade de países altamente endividados em instaurarem políticas expansionistas. $\mathrm{Na}$ regressão que utiliza a taxa de juros real o sinal foi oposto (também não significativo), possivelmente enviesado pela omissão da taxa de juros nominal.

A razão crédito privado/PIB, embora tenha apresentado coeficientes negativos - em linha com o que o referencial teórico apresentou sobre a transmissão da crise via o mercado de crédito - estes não se mostraram significativos estatisticamente, diferentemente do trabalho de Lane e Milesi-Ferretti (2010). A variação do crédito privado/PIB também apresentou coeficientes negativos, em linha com os trabalhos apresentados que expõe uma maior severidade nos países que tiveram um rápido crescimento do mercado de crédito em função da reversão dessa tendência - dado ambiente de incerteza e baixa liquidez - contudo, os coeficientes não apresentaram significância estatística.

A variável volatilidade do PIB obteve coeficientes positivos nas duas especificações significativos em ambas. O crescimento médio do PIB (1990-2007) e a dummy para exportadores de petróleo e a constante da regressão não se mostraram estatisticamente significativos nas duas regressões. Tanto a variável PIB per capita, como o crescimento do PIB per capita registraram coeficientes negativos - contudo apenas a variável de crescimento 
PIB per capita apresentou significância estatística - indicando maior severidade da crise financeira nos países que mais se desenvolveram entre 2003 e 2007.

A variável de regime cambial apresentou coeficientes negativos, mas não significativos estatisticamente. O resultado indica que países com regimes mais flexíveis da taxa de câmbio foram mais afetados pela da crise de 2008, tudo mais constante. Uma hipótese para essa evidência seria que os países que receberam uma grande quantidade de capital (em função do Global Saving Glut) no momento da crise observaram o êxodo dos recursos, depreciando suas moedas e impactando negativamente o produto da sua economia, seja pela diminuição no resultado comercial (assumindo a dinâmica da curva $J$ e a condição de Marshall-Lerner sobre exportações/importações e taxa de câmbio) ou pela contração nos investimentos dada uma possível maior exposição cambial por parte das empresas nãofinanceiras.

A variável de abertura comercial apresentou coeficientes negativos e estatisticamente significativos, em linha com a idéia de que uma maior integração comercial poderia gerar uma sensibilidade maior às recessões nos parceiros comerciais, resultando em uma crise mais severa, tudo mais constante. A relação conta corrente/PIB apresentou coeficientes estatisticamente significativos positivos. Possivelmente, países que incorrem em resultados comerciais positivos exportam poupança, e em momentos de crise o capital ao regressar ao país de origem ajuda o ambiente de liquidez, minimizando a severidade da crise financeira. A tabela 2 sintetiza o resultado das estimações.

No que diz respeito à adequação do método escolhido, as regressões se enquadram no Teste de Ramsey ${ }^{12}$. Contudo, pode-se imaginar que a severidade da crise financeira e a capacidade de resposta de política econômica possam estar relacionadas com uma outra variável. Parte do ambiente de liquidez no qual se desenvolveu a crise financeira tem origem no fluxo de capitais proveniente do Global Saving Glut, e este pode ter sido direcionado com base na classificação dos países em relação à dívida soberana dos mesmos (KIM e WU, 2008).

Embora a atribuição de grau de investimento a um país signifique que o mesmo se mostre mais preparado para uma situação de aversão de risco e a choques exógenos, o fato da classificação de dívida dos países direcionar o fluxo de capitais pode fazer com que um país investment grade, por ter recebido uma maior quantidade de capital, possa estar mais

\footnotetext{
${ }^{12}$ As regressões apresentaram probabilidae F de 0,4413 e 0,3977, aceitando a hipótese nula de que o modelo não apresenta variáveis omitidas.
} 
vulnerável ao êxodo dos recursos do que um país classificado como speculative grade, que teria recebido uma quantidade menor de recursos e, portanto, seria menos "dependente" dos recursos externos, portanto, menos sensível ao movimento de evasão do capital.

Ao analisar os dados dos 100 países da amostra que apresentavam classificação da sua dívida soberana no final de $2007^{13}$, constatou-se que o comportamento do produto nos países classificados como investment grade apresentou variação média negativa de $0,52 \%$ e desviopadrão de 3,34\%. Já os países considerados como speculative grade apresentaram uma variação média positiva de 3,30\% e um desvio-padrão de 3,47\%. A taxa de juros nominal média dos países investment grade em 2007 de 5,09\% ao ano, com desvio-padrão de 2,51\%. Nos países speculative grade a taxa média de 7,87\% e o desvio-padrão de 5,23\%.

A taxa de juros real média em 2007 nos países investment grade foi de 1,51\% ao ano, com desvio-padrão de $2,42 \%$. Os países speculative grade apresentaram uma média de $0,84 \%$ e um desvio-padrão de 4,47\%. Em relação à variável de política fiscal, o resultado do governo em relação ao PIB médio dos países investment grade foi de 2,23\% - desvio-padrão de $6,81 \%$. Já os países speculative grade apresentaram um resultado médio negativo de $0,61 \%$, com um desvio-padrão de 4,12\%.

Para controlar o efeito do fluxo de capital anterior à crise nas regressões, foi adicionada às especificações supracitadas uma variável dummy de investment grade. Foi atribuído o valor "1" para os países classificados como investment grade e "0" para os países classificados como speculative grade. As regressões estimadas seguem o mesmo padrão das estimações feitas sem a dummy de investment grade - regressões (3) e (4) contêm a variação do PIB como variável endógena e diferem apenas na variável de política monetária, taxa de juros nominal e taxa de juros real, respectivamente. Com a inclusão da dummy observou-se que o coeficiente da variável de política monetária, a taxa de juros nominal, foi positivo - em linha com a hipótese da pesquisa em relação à capacidade de resposta da política monetária e com as estimações prévias - e estatisticamente significativo. A taxa de juros real apresentou um coeficiente positivo, entretanto, não foi estatisticamente significativo.

A variável de política fiscal apresentou coeficientes negativos estatisticamente significativos, assim como anteriormente, contrariando a hipótese do trabalho. A variável de interação fiscal embora tenha apresentado coeficientes positivos - em linha com a hipótese do trabalho - com a inclusão da dummy de investment grade os parâmetros não foram

\footnotetext{
${ }^{13}$ Com base no rating da dívida soberana do país em 2007 atribuído pela Fitch Ratings. A relação dos países da amostra com suas respectivas notas, bem como a escala utilizada pela agência classificadora de risco encontra-se no quadro 3.
} 
estatisticamente positivos. A dummy de investment grade apresentou coeficientes negativos estatisticamente significativo apenas na regressão com a taxa de juros real - indicando que países que tinham a classificação de grau de investimento no final de 2007 tiveram uma crise mais severa, em média, do que países cuja classificação era de grau especulativo, em linha com a hipótese apresentada. Destaca-se o valor dos coeficientes. Na regressão (4), só o "selo" investment grade representou uma queda de 3,5\% no PIB, em média, tudo mais constante.

A razão crédito privado/PIB apresentou coeficientes positivos (diferentemente das estimações iniciais), contudo, não foram estatisticamente significativos. A variável volatilidade do PIB teve coeficientes positivos não significativos estatisticamente. $O$ crescimento médio do PIB também apresentou coeficientes positivos e não significativos estatisticamente. A dummy para países exportadores de petróleo teve coeficientes positivos, todavia, não se mostraram estatisticamente significativos.

A variável PIB per capita que nas primeiras estimações havia apresentado coeficientes estatisticamente significativos, com a inclusão da dummy de investment grade registrou coeficientes negativos, mas não significativos. O crescimento médio do PIB per capita continuou com coeficientes negativos e estatisticamente significativos. Países que apresentaram um crescimento acelerado (possivelmente acima de um nível sustentável) apresentaram uma crise mais severa, tudo mais constante.

A razão Conta Corrente/PIB apresentou coeficientes positivos assim como nas estimações sem a variável de investment grade, contudo, não foram estatisticamente significativos. As variáveis de regime cambial e de abertura comercial não se mostraram estatisticamente significativas. A tabela 3 sintetiza o resultado das estimações com a dummy de investment grade.

A inclusão da nova variável fez com que o tamanho da amostra diminuísse em função da disponibilidade de ratings, mas os modelos continuam bem especificados ${ }^{14}$. Embora o objetivo do trabalho não seja avaliar as causas da crise financeira, e sim achar relações entre a capacidade de política econômica e a severidade da crise financeira, no que diz respeito ao poder explicativo das regressões medido pela estatística $\mathrm{R}^{2}$ ajustado, a inclusão da dummy de investment grade trouxe melhoras às estimações. Por exemplo, analisando a regressão que contempla a taxa de juros nominal apresentou nas primeiras estimações um $\mathrm{R}^{2}$ ajustado de

\footnotetext{
${ }^{14}$ As regressões (3) e (4) apresentaram probabilidade $\mathrm{F}$ de 0,7222 e 0,2822, respectivamente, aceitando a hipótese nula de que o modelo não apresenta variáveis omitidas no teste de Ramsey.
} 
0,3413. A adição da nova variável à especificação registrou um $\mathbf{R}^{2}$ ajustado de 0,3934 . Nas estimativas com a taxa de juros real, o $\mathrm{R}^{2}$ ajustado aumentou de 0,3354 para 0,3855 .

A pesquisa conseguiu identificar correlações importantes entre o grau de severidade da crise financeira internacional e o estado dos instrumentos de política monetária e fiscal antes do evento. Países com maiores taxas de juros registraram uma crise menos severa, tudo mais constante, em linha com a hipótese apresentada. Vale ressalvar, no entanto, que a heterogeneidade dos países da amostra pode fazer com que o modelo não capte, necessariamente, a capacidade de resposta como fator que minimiza a severidade da crise. Embora a intuição econômica e as estimativas das regressões apontem nessa direção (que o modelo esteja bem especificado de acordo com o teste de Ramsey), a grande diferença entre os países dificulta o controle por outros fatores relacionados.

No âmbito fiscal, embora a variável de interesse - resultado do governo central tenha apresentado um coeficiente negativo, contrariando a hipótese do trabalho em função de possíveis limites aos estímulos através de gastos do governo já discutidos, a variável de interação fiscal que condiciona a capacidade de reposta da política fiscal não apenas ao fluxo no ano anterior à crise, mas também ao estoque de dívida do país mostrou que uma situação mais sólida foi fator relevante para minimizar os efeitos da crise financeira no primeiro ano do evento.

O trabalho está em linha com as novas recomendações de políticas econômicas ${ }^{15}$, focadas na preocupação em manter níveis baixos de juros, mas não excessivamente baixos, nem por muito tempo, e na utilização de estímulos fiscais anti-cíclicos de curto prazo quando necessário, quando há clara falta de demanda agregada e os intrstumentos tradicionais de política monetária não forem suficientes para restaurar o pleno emprego.

\section{Conclusão}

Após um breve estudo sobre a crise de 2008, pôde-se compreender porque o episódio configura a maior crise financeira desde a Grande Deepressão. Isoladamente, contrações no mercado de crédito, evolução acentuada dos preços dos imóveis e o comportamento do setor bancário são, em geral, responsáveis por episódios recessivos mais severos. A intensidade aumenta quando combinados. A crise de 2008 apoia-se nesses três pilares e, portanto os seus desdobramentos e as proporções alcançadas não poderiam ser diferentes.

\footnotetext{
${ }^{15}$ BLANCHARD et al, 2010
} 
Durante o processo de recuperação, as diferenças históricas entre países desenvolvidos e emergentes podem configurar uma importante base para a compreensão dos acontecimentos recentes e dos que estão por vir. A capacidade de resposta da política econômica se mostrou fator relevante para minimizar os efeitos da crise financeira no seu primeiro ano. Em relação à política monetária, países que experimentaram taxas de juros maiores no final de 2007 apresentaram um maior espaço para implementação de políticas expansionistas e tiveram uma crise menos severa. Taxas de juros nominais mais altas podem tornar mais difícil que um país seja preso na armadilha da liquidez. Assim, os instrumentos usuais disponíveis aos bancos centrais seriam suficientes (ou pelo menos mais potentes) não apenas para suavizar os ciclos de negócio, mas para combater crises não tão severas, além de proporcionar maior capacidade de resposta à crises financeiras que, em geral, têm não somento um desenvolvimento hermético, como também a sua resolução.

$\mathrm{Na}$ análise da política fiscal, embora os coeficientes do resultado do governo central confrontem a hipótese apresentada, a variável de interação fiscal que capta a gestão feita não apenas considerando o fluxo no ano de 2007, mas também o endividamento bruto público aponta na direção da hipótese do trabalho de que países que vivenciam uma melhor situação fiscal sentiram menos os efeitos da crise financeira através da variação do produto no primeiro ano do evento, pois apresentavam um maior espaço para instaurar estímulos fiscais de curto prazo.

Com a inclusão de uma nova variável para capturar o efeito do direcionamento dos recursos pré-crise e possivelmente os impactos da reversão desse movimento durante o evento analisado, os resultados apontam para uma crise mais severa nas economias mais desenvolvidas que apresentavam a qualificação de grau de investimento - essa conclusão é reforçada pelo valor dos coeficientes da dummy de investment grade. O controle pelo PIB per capita e pelo crescimento do mesmo também apontou para essa direção.

No que tange as implicações de política econômica dos resultados obtidos, a gestão de política monetária além de objetivar a estabilidade de preços e possivelmente o pleno emprego, deve também considerar a recorrência de crises e recessões, e a capacidade de resposta ganha importância nesses eventos, confrontando práticas de taxas de juros muito baixas por muito tempo.

No âmbito fiscal a gestão concomitante do resultado do governo central e do endividamento do país aponta para uma maior segurança macroeconômica durante episódios como o registrado em 2008. Estímulos keynesianos de curto prazo são importantes e a 
capacidade de implementá-los se mostrou fator relevante para minimizar a severidade da crise de 2008, mas está condicionada às práticas dos policymakers durante momentos de moderação.

Em suma, poderia ter sido diferente? Sim, se os bancos centrais não tivessem mantido juros tão baixos por tanto tempo, aceitando um menor ritmo de crescimento no período précrise, mas com maior capacidade de resposta quando os ventos mudassem.

\section{Referências}

BANCO MUNDIAL. Disponível em: data.worldbank.org. Acesso em 19 de outubro de 2009.

BARRO, Robert J. Determinants of economic growth: a cross-country empirical study. National Bureau of Economic Research, 1996.

BERNANKE, Ben S. The Global Saving Glut and the U.S. Current Account Deficit, Discurso na Sandridge Lecture, Virginia Association of Economists, Richmond, Virginia, 2005.

BLANCHARD, Olivier J. et al. The Initial Impact of the Crisis on Emerging Market Countries [with Comments and Discussion]. Brookings papers on economic activity, p. 263-323, 2010.

BORDO, Michael D.; HAUBRICH, Joseph G. Deep recessions, fast recoveries, and financial crises: Evidence from the American record. National Bureau of Economic Research, 2012.

BUBULA, Andrea; OTKER-ROBE, Inci. Are Pegged Exchange Rate Regimes More Crisis Prone?. IMF Working Paper 03/223, novembro de 2003.

CALOMIRIS, Charles W.. The Subprime Turmoil: What's Old, What's New and What's Next. Federal Reserve Bank of Kansas City's Symposium, Maintaining Stability in a Changing Financial System, outubro de 2008.

CLAESSENS, Stijn; KOSE, Ayhan M.; TERRONES, Marco E. What happens during recessions, crunches, and busts? IMF Working Paper, Agosto, 2008.

CLAESSENS, Stijn; PAZARBASIOGIU, Ceyla; LAEVEN, Luc; DOBLER, Marc; VALENCIA, Fabian; NEDELESCU, Oana; SEAL, Katharine. Crisis Management and Resolution: Early Lessons from the Financial Crisis. IMF Staff Discution Note, Março, 2009.

EICHENGREEN, Barry. International Financial Crises: Is the Problem

Growing? Jahrbuch für Wirtschaftsgeschichte/Economic History Yearbook43.1 (2002): 89104.

FITCH RATINGS, Acesso 10 de julho de 2011 em www.fitchratings.com 
FUNDO MONETÁRIO INTERNACIONAL, Acesso 19 de outubro de 2010, em www.imf.org.

IMBS, Jean. The First Global Recession in Decades. HEC Lausanne Swiss Finance Institute and CEPR, dezembro de 2009.

KAMIN, Steven B.; DEMARCO, Laurie Pounder. How Did a Domestic Housing Slump Turn into a Global Financial Crisis? Board of Governors of the Federal Reserve System, International Finance Discussion Papers, janeiro de 2010.

KIYOTAKI, Nobuhiro; MOORE, John. Credit Cycles. The Journal of Political Economy. vol. 105, p. 211 - 248. Abril, 1997. crossref http://dx.doi.org/10.1086/262072

KIM, Suk-Joong,; WU, Eliza. Sovereign credit ratings, capital flows and financial sector development in emerging markets. Emerging Markets Review, Vol. 9, No. 1, pp. 17-39,

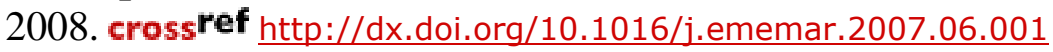

LAEVEN, Luc; VALENCIA, Fabian. Resolution of Banking Crises: The Good, the Bad, and the Ugly. IMF Working Paper, junho de 2010.

LANE, Philip R.; MILESI-FERRETI, Gian Maria. The Cross-Country Incidence of the Global Crisis. IMF Working Paper, julho 2010.

REINHART, Carmen M.; ROGOFF, Kenneth S.. Is the U.S. Sub-prime financial crisis so different? An international historical comparison. NBER Working Paper, 2008

The Aftermath of Financial Crisis. NBER Working Paper, janeiro de 2009

REINHART, Carmen M.; ROGOFF, Kenneth S.; SAVASTANO, Miguel A.. Debt

Intolerance. NBER Working Paper, agosto de 2003.

REINHART, Carmen M.; REINHART, Vincent R.. After the Fall. NBER Working Paper Series, setembro 2010

ROSE, Andrew K.; SPIEGEL, Mark M. The Causes and Consequences of the 2008 Crisis: Early Warning. Global Journal of Economics. NBER Working Paper Series, setembro 2009.

STANDARD \& POOR'S, acesso 10 de julho de 2011, em http://www2.standardandpoors.com/

TAYLOR, John B. The Financial Crisis and the Policy Responses: An Empirical Analysis of What Went Wrong. NBER Working Paper No 14631, janeiro de 2009. 


\section{Apêndice - Variáveis utilizadas na análise econométrica}

Quadro 1: Descrição das variáveis

\begin{tabular}{|c|c|c|}
\hline Variável & Descrição & Fonte \\
\hline \multicolumn{3}{|c|}{ Variável Dependente } \\
\hline Variação PIB (2009/2008) & $\begin{array}{l}\text { Variação percentual do valor } \\
\text { do PIB a preços constantes } \\
\text { em } 2009 \text { frente ao valor } \\
\text { auferido em } 2008 .\end{array}$ & $\begin{array}{c}\text { World Economic Outlook } \\
\text { (WEO), FMI, Abril de } 2011 .\end{array}$ \\
\hline \multicolumn{3}{|c|}{ Variáveis explicativas } \\
\hline Taxa de Juros Nominal & $\begin{array}{c}\text { Taxa de juros nominal } \\
\text { (Money Market) do país em } \\
\text { 2007. Para os países que não } \\
\text { possuíam esse tipo de taxa, } \\
\text { foram feitas substituições } \\
\text { conforme quadro } 4 .\end{array}$ & IFS, FMI Outubro de 2010. \\
\hline Taxa de Juros Real & $\begin{array}{c}\text { Taxa de juros nominal } \\
\text { deflacionada pelo CPI do } \\
\text { país. }\end{array}$ & $\begin{array}{l}\text { O CPI foi obtido no Banco } \\
\text { Mundial, dados de } 2007 \text { e o } \\
\text { cálculo foi feito pelo autor. }\end{array}$ \\
\hline Resultado Fiscal & $\begin{array}{c}\text { Resultado Fiscal do Governo } \\
\text { Central em percentual do PIB } \\
\text { em } 2007 .\end{array}$ & $\begin{array}{l}\text { WEO, Outubro de } 2010 \text {, } \\
\text { código: GGXCNL_NGDP }\end{array}$ \\
\hline Dívida/PIB & $\begin{array}{l}\text { Dívida bruta sobre o PIB em } \\
2007\end{array}$ & $\begin{array}{l}\text { World Economic Outlook, } \\
\text { FMI, Abril de } 2011 .\end{array}$ \\
\hline \multicolumn{3}{|c|}{ Variáveis de Controle } \\
\hline Crédito Privado/PIB & $\begin{array}{c}\text { Razão crédito privado sobre } \\
\text { o PIB em } 2007 .\end{array}$ & $\begin{array}{l}\text { Base de dados do artigo "The } \\
\text { Cross-Country Incidence of } \\
\text { the Global Crisis". }\end{array}$ \\
\hline $\begin{array}{c}\text { Variação do Crédito } \\
\text { Privado/PIB: }\end{array}$ & $\begin{array}{l}\text { Variação da razão } \\
\text { crédito/PIB de } 3003 \text { a } 2007 .\end{array}$ & $\begin{array}{l}\text { Base de dados do artigo "The } \\
\text { Cross-Country Incidence of } \\
\text { the Global Crisis". }\end{array}$ \\
\hline
\end{tabular}

Fonte: Elaboração do autor 
Quadro 1 (cont.): Descrição das variáveis

\begin{tabular}{|c|c|c|}
\hline Volatilidade do PIB & $\begin{array}{l}\text { Desvio padrão do logarítimo } \\
\text { natural da razão PIB no } \\
\text { instante t sobre PIB em t-1. }\end{array}$ & $\begin{array}{l}\text { Base de dados do artigo "The } \\
\text { Cross-Country Incidence of } \\
\text { the Global Crisis". }\end{array}$ \\
\hline Crescimento Médio do PIB & $\begin{array}{l}\text { Crescimento médio do PIB } \\
\text { entre } 1990 \text { e } 2007 .\end{array}$ & $\begin{array}{l}\text { Base de dados do artigo "The } \\
\text { Cross-Country Incidence of } \\
\text { the Global Crisis". }\end{array}$ \\
\hline $\begin{array}{c}\text { Dummy (Exportadores de } \\
\text { Petróleo) }\end{array}$ & $\begin{array}{l}\text { Dummy com valor } 1 \text { para os } \\
\text { países exportadores de } \\
\text { petróleo e " } 0 \text { " para os } \\
\text { demais. }\end{array}$ & $\begin{array}{l}\text { Base de dados do artigo "The } \\
\text { Cross-Country Incidence of } \\
\text { the Global Crisis". }\end{array}$ \\
\hline Dummy Investment Grade & $\begin{array}{l}\text { Dummy com valor } 1 \text { para os } \\
\text { países com grau de } \\
\text { investimento e " } 0 \text { " para os } \\
\text { demais. }\end{array}$ & Fitch Ratings. \\
\hline PIB per capita & PIB per capita em 2007 & $\begin{array}{l}\text { Base de dados do artigo "The } \\
\text { Cross-Country Incidence of } \\
\text { the Global Crisis". }\end{array}$ \\
\hline $\begin{array}{c}\text { Crescimento do PIB per } \\
\text { capita }\end{array}$ & $\begin{array}{l}\text { Variação percentual do PIB } \\
\text { per capita em de } 2003 \text { a } \\
2007 .\end{array}$ & $\begin{array}{l}\text { Cálculo do autor com base } \\
\text { nos dados do World } \\
\text { Economic Outlook, Abril de } \\
2011 .\end{array}$ \\
\hline Conta Corrente/PIB & $\begin{array}{l}\text { Valor da conta corrente sobre } \\
\qquad \text { o PIB em } 2007 .\end{array}$ & $\begin{array}{l}\text { Base de dados do artigo "The } \\
\text { Cross-Country Incidence of } \\
\text { the Global Crisis". }\end{array}$ \\
\hline Regime Cambial de facto & $\begin{array}{l}\text { Regime cambial de } 2007 \\
\text { com base na metodologia } \\
\text { proposta por Reinhart e } \\
\text { Rogoff. }\end{array}$ & Reinhart e Rogoff (2007) \\
\hline Abertura Comercial & $\begin{array}{c}\text { Exportações mais } \\
\text { Importações sobre o PIB. }\end{array}$ & $\begin{array}{l}\text { WDI, Outubro de 2010, } \\
\text { código: NE.TRD.GNFS.ZS }\end{array}$ \\
\hline
\end{tabular}

Fonte: Elaboração do autor 
Quadro 2: Relação de países da amostra completa

\begin{tabular}{|c|c|c|}
\hline Albania & Greece & Pakistan \\
\hline Algeria & Grenada & Panama \\
\hline Angola & Guatemala & Papua New Guinea \\
\hline Antigua and Barbuda & Guinea & Paraguay \\
\hline Argentina & Guinea-Bissau & Peru \\
\hline Armenia & Guyana & Philippines \\
\hline Australia & Haiti & Poland \\
\hline Austria & Honduras & Portugal \\
\hline Azerbaijan & Hong Kong SAR & Qatar \\
\hline Bahrain & Hungary & Republic of Congo \\
\hline Bangladesh & Iceland & Republic of Yemen \\
\hline Barbados & India & Romania \\
\hline Belarus & Indonesia & Russia \\
\hline Belgium & Iraq & Rwanda \\
\hline Belize & Ireland & Samoa \\
\hline Benin & Islamic Republic of Afghanistan & São Tomé and Príncipe \\
\hline Bhutan & Islamic Republic of Iran & Saudi Arabia \\
\hline Bolivia & Israel & Senegal \\
\hline Bosnia and Herzegovina & Italy & Serbia \\
\hline Botswana & Jamaica & Seychelles \\
\hline Brazil & Japan & Sierra Leone \\
\hline Brunei Darussalam & Jordan & Singapore \\
\hline Bulgaria & Kazakhstan & Slovak Republic \\
\hline Burkina Faso & Kenya & Slovenia \\
\hline Burundi & Kiribati & Solomon Islands \\
\hline Cambodia & Korea & South Africa \\
\hline Cameroon & Kosovo & Spain \\
\hline Canada & Kuwait & Sri Lanka \\
\hline Cape Verde & Kyrgyz Republic & St. Kitts and Nevis \\
\hline Central African Republic & Lao People's Democratic Republic & St. Lucia \\
\hline Chad & Latvia & St. Vincent and the Grenadines \\
\hline Chile & Lebanon & Sudan \\
\hline China & Lesotho & Suriname \\
\hline Colombia & Liberia & Swaziland \\
\hline Comoros & Libya & Sweden \\
\hline Costa Rica & Lithuania & Switzerland \\
\hline Côte d'Ivoire & Luxembourg & Syrian Arab Republic \\
\hline Croatia & Madagascar & Taiwan Province of China \\
\hline Cyprus & Malawi & Tajikistan \\
\hline Czech Republic & Malaysia & Tanzania \\
\hline Democratic Republic of Congo & Maldives & Thailand \\
\hline Democratic Republic of Timor-Leste & Mali & The Bahamas \\
\hline Denmark & Malta & The Gambia \\
\hline Djibouti & Mauritania & Togo \\
\hline Dominica & Mauritius & Tonga \\
\hline Dominican Republic & Mexico & Trinidad and Tobago \\
\hline Ecuador & Moldova & Tunisia \\
\hline Egypt & Mongolia & Turkey \\
\hline El Salvador & Montenegro & Turkmenistan \\
\hline Equatorial Guinea & Morocco & Uganda \\
\hline Eritrea & Mozambique & Ukraine \\
\hline Estonia & Myanmar & United Arab Emirates \\
\hline Ethiopia & Namibia & United Kingdom \\
\hline Fiji & Nepal & United States \\
\hline Finland & Netherlands & Uruguay \\
\hline Former Yugoslav Republic of Macedonia & New Zealand & Uzbekistan \\
\hline France & Nicaragua & Vanuatu \\
\hline Gabon & Niger & Venezuela \\
\hline Georgia & Nigeria & Vietnam \\
\hline Germany & Norway & Zambia \\
\hline Ghana & Oman & Zimbabwe \\
\hline
\end{tabular}

Fonte: Elaboração do autor 
Tabela 2: Resultados das Regressões

\begin{tabular}{|c|c|c|}
\hline & (1) & (2) \\
\hline \multirow{2}{*}{ Taxa de Juros Nominal } & 0,201 & \\
\hline & {$[0.1700]$} & \\
\hline \multirow{2}{*}{ Taxa de Juros Real } & & $-0,048$ \\
\hline & & {$[0.7038]$} \\
\hline \multirow{2}{*}{ Resultado Fiscal } & $-0,474$ & $-0,533$ \\
\hline & {$[0.0006]^{* * *}$} & {$[0.0002]^{* * *}$} \\
\hline \multirow{2}{*}{ Dívida/PIB } & $-0,313$ & 0,011 \\
\hline & [0.828] & [0.994] \\
\hline \multirow{2}{*}{ Interação $_{(\text {Resultado Fiscal * Dívida/PIB) }}$} & 0,555 & 0,711 \\
\hline & {$[0.0109]^{* *}$} & {$[0.0015]^{* * *}$} \\
\hline \multirow{2}{*}{ Crédito Privado/PIB } & $-0,757$ & $-1,205$ \\
\hline & {$[0.5656]$} & {$[0.3617]$} \\
\hline \multirow{2}{*}{$\Delta$ Crédito Privado/PIB ${ }_{(2003-2007)}$} & $-2,163$ & $-0,694$ \\
\hline & {$[0.4014]$} & {$[0.7868]$} \\
\hline \multirow{2}{*}{ Volatilidade do PIB $_{(1990-2007)}$} & 0,432 & 0,516 \\
\hline & {$[0.0227]^{* *}$} & {$[0.0054]^{* * *}$} \\
\hline \multirow{2}{*}{ Crescimento Médio do PIB $_{(1990-2007)}$} & 0,379 & 0,374 \\
\hline & {$[0.1710]$} & [0.1899] \\
\hline \multirow{2}{*}{ Dummy (Exportadores de Petróleo) } & 2,469 & 2,752 \\
\hline & {$[0.2202]$} & {$[0.1806]$} \\
\hline \multirow{2}{*}{ PIB per capita } & 0,000 & 0,000 \\
\hline & {$[0.3035]$} & [0.3023] \\
\hline \multirow{2}{*}{ Crescimento do PIB per capita $_{(2004-2007)}$} & $-4,959$ & $-5,354$ \\
\hline & {$[0.005]^{* * *}$} & {$[0.003]^{* * *}$} \\
\hline \multirow{2}{*}{ Conta Corrente/PIB } & 14,327 & 13,664 \\
\hline & {$[0.0005]^{* * *}$} & {$[0.0009]^{* * *}$} \\
\hline \multirow{2}{*}{ Regime Cambial } & $-0,632$ & $-0,520$ \\
\hline & [0.1944] & [0.2964] \\
\hline \multirow{2}{*}{ Abertura Comercial } & $-0,012$ & $-0,014$ \\
\hline & {$[0.0655]^{*}$} & {$[0.0440]^{* *}$} \\
\hline \multirow{2}{*}{ Constante } & 2,760 & 3,920 \\
\hline & {$[0.1547]$} & {$[0.0454]^{* *}$} \\
\hline Observações & 114 & 113 \\
\hline $\mathrm{R}^{2}$ Ajustado & 0,3413 & 0,3354 \\
\hline Prob. F & 0,0000 & 0,0000 \\
\hline
\end{tabular}

[]: p-valor; $* * *, * *$ e $*$ : significativo a $1 \%, 5 \%$ e $10 \%$, respectivamente

Fonte: Elaboração do Autor com base nos resultados obtidos através do Stata 9.0 
Tabela 3: Resultados das Regressões com a dummy de investment grade

\begin{tabular}{|c|c|c|}
\hline & (3) & (4) \\
\hline Taxa de Juros Nominal & $\begin{array}{c}0,366 \\
{[0.0871]^{*}}\end{array}$ & \\
\hline Taxa de Juros Real & & $\begin{array}{c}0,280 \\
{[0.1330]}\end{array}$ \\
\hline Resultado Fiscal & $\begin{array}{c}-0,225 \\
{[0.0996]^{*}}\end{array}$ & $\begin{array}{c}-0,256 \\
{[0.0532]^{*}}\end{array}$ \\
\hline Dívida/PIB & $\begin{array}{c}-0,492 \\
{[0.782]}\end{array}$ & $\begin{array}{c}-0,070 \\
{[0.972]}\end{array}$ \\
\hline Interação $_{(\text {Resultado Fiscal * Dívida/PIB) }}$ & $\begin{array}{c}0,309 \\
{[0.2903]}\end{array}$ & $\begin{array}{c}0,305 \\
{[0.3336]}\end{array}$ \\
\hline Crédito Privado/PIB & $\begin{array}{c}1,386 \\
{[0.3759]}\end{array}$ & $\begin{array}{c}1,055 \\
{[0.4933]}\end{array}$ \\
\hline$\Delta$ Crédito Privado/PIB $(2003-2007)$ & $\begin{array}{c}-4,317 \\
{[0.1134]}\end{array}$ & $\begin{array}{c}-2,907 \\
{[0.2508]}\end{array}$ \\
\hline Volatilidade do $\mathrm{PIB}_{(1990-2007)}$ & $\begin{array}{c}0,069 \\
{[0.7246]}\end{array}$ & $\begin{array}{c}0,194 \\
{[0.3263]}\end{array}$ \\
\hline Crescimento Médio do $\mathrm{PIB}_{(1990-2007)}$ & $\begin{array}{c}0,349 \\
{[0.3183]}\end{array}$ & $\begin{array}{c}0,291 \\
{[0.4307]}\end{array}$ \\
\hline Dummy (Exportadores de Petróleo) & $\begin{array}{c}2,118 \\
{[0.4767]}\end{array}$ & $\begin{array}{c}3,034 \\
{[0.3707]}\end{array}$ \\
\hline PIB per capita & $\begin{array}{c}0,000 \\
{[0.2355]}\end{array}$ & $\begin{array}{c}0,000 \\
{[0.1968]}\end{array}$ \\
\hline Crescimento do PIB per capita $(2004-2007)$ & $\begin{array}{c}-5,148 \\
{[0.016]^{* *}}\end{array}$ & $\begin{array}{c}-4,903 \\
{[0.034]^{* *}}\end{array}$ \\
\hline Conta Corrente/PIB & $\begin{array}{c}10,079 \\
{[0.1646]}\end{array}$ & $\begin{array}{c}7,473 \\
{[0.3443]}\end{array}$ \\
\hline Regime Cambial & $\begin{array}{c}-0,180 \\
{[0.7331]}\end{array}$ & $\begin{array}{c}0,035 \\
{[0.9461]}\end{array}$ \\
\hline Abertura Comercial & $\begin{array}{c}-0,008 \\
{[0.2915]}\end{array}$ & $\begin{array}{c}-0,004 \\
{[0.6202]}\end{array}$ \\
\hline Dummy (Investment Grade) & $\begin{array}{l}-2,632 \\
{[0.112]}\end{array}$ & $\begin{array}{c}-3,541 \\
{[0.035]^{* *}}\end{array}$ \\
\hline Constante & $\begin{array}{c}1,796 \\
{[0.4400]}\end{array}$ & $\begin{array}{c}2,833 \\
{[0.2255]}\end{array}$ \\
\hline Observações & 83 & 82 \\
\hline $\mathrm{R}^{2}$ Ajustado & 0,3934 & 0,3855 \\
\hline Prob. F & 0,0000 & 0,0000 \\
\hline
\end{tabular}

[]: p-valor; $* * *, * *$ e $*$ : significativo a $1 \%, 5 \%$ e $10 \%$, respectivamente

Fonte: Elaboração do Autor com base nos resultados obtidos através do Stata 9.0 Revista Economia Ensaios, Uberlândia (MG), 30 (1), p. 66-94, Jul./Dez. 2015 
Tabela 4: Classificação da Fitch Ratings

\begin{tabular}{cccc} 
Rating & Classificação & Rating & Classificação \\
\hline \hline AAA & Investment Grade & BB & Speculative Grade \\
AA+ & Investment Grade & BB- & Speculative Grade \\
AA & Investment Grade & B+ & Speculative Grade \\
AA- & Investment Grade & B & Speculative Grade \\
A+ & Investment Grade & B- & Speculative Grade \\
A & Investment Grade & CCC+ & Speculative Grade \\
A- & Investment Grade & CCC & Speculative Grade \\
BBB+ & Investment Grade & CCC- & Speculative Grade \\
BBB & Investment Grade & CC & Speculative Grade \\
BBB- & Investment Grade & C & Speculative Grade \\
BB+ & Speculative Grade & D & Speculative Grade \\
\hline
\end{tabular}

Fonte: www.fitchratings.com; Elaboração do autor

Quadro 3: Rating da Dívida Soberana no final de 2007

\begin{tabular}{|c|c|c|c|c|c|}
\hline País & Data da atribuição do Rating & Rating & País & Data da atribuição do Rating & Rating \\
\hline Abu Dhabi & 2 jul 2007 & AA & Finland & 21 set 2000 & AAA \\
\hline Argentina & 1 ago 2006 & $\mathrm{RD}$ & France & 21 set 2000 & AAA \\
\hline Armenia & 1 mai 2007 & BB- & Gabon & 29 out 2007 & BB- \\
\hline Aruba & 8 jun 2006 & BBB & Georgia & 18 jul 2007 & BB- \\
\hline Australia & 3 fev 2003 & $\mathrm{AA}+$ & Germany & 21 set 2000 & AAA \\
\hline Austria & 21 set 2000 & AAA & Ghana & 1 fev 2006 & $\mathrm{~B}+$ \\
\hline Azerbaijan & 5 fev 2007 & $\mathrm{BB}+$ & Greece & 5 mar 2007 & $\mathrm{~A}$ \\
\hline Bahrain & 24 ago 2007 & A & Guatemala & 22 fev 2006 & $\mathrm{BB}+$ \\
\hline Belgium & 2 mai 2006 & $\mathrm{AA}+$ & Hong Kong & 12 jul 2007 & AA \\
\hline Benin & 15 set 2004 & B & Hungary & 5 nov 2007 & $\mathrm{BBB}+$ \\
\hline Bermuda & 11 ago 2006 & $\mathrm{AA}+$ & Iceland & 15 mar 2007 & $\mathrm{~A}+$ \\
\hline Bolivia & 27 jul 2007 & B- & India & 1 ago 2006 & BBB- \\
\hline Brazil & 9 mai 2007 & $\mathrm{BB}+$ & Indonesia & 28 jan 2007 & BB- \\
\hline Bulgaria & 17 ago 2005 & BBB & Iran & 24 abr 2006 & $\mathrm{~B}+$ \\
\hline Cameroon & 6 mar 2007 & B & Ireland & 21 set 2000 & AAA \\
\hline Canada & 12 ago 2004 & AAA & Israel & $18 \mathrm{dez} 2006$ & A- \\
\hline Cape Verde & 15 ago 2003 & $\mathrm{~B}+$ & Italy & 19 out 2006 & AA- \\
\hline Chile & 15 mai 2007 & $\mathrm{~A}$ & Jamaica & 29 ago 2006 & $\mathrm{~B}+$ \\
\hline China & 6 nov 2007 & $A+$ & Japan & 9 mai 2005 & AA \\
\hline Colombia & 21 jun 2007 & $\mathrm{BB}+$ & Kazakhstan & $17 \operatorname{dez} 2007$ & BBB \\
\hline Costa Rica & 4 out 2006 & BB & Kenya & $12 \operatorname{dez} 2007$ & $\mathrm{~B}+$ \\
\hline Croatia & 6 jul 2005 & BBB- & Korea & 23 out 2005 & $A+$ \\
\hline Cyprus & 12 jul 2007 & AA- & Kuwait & 10 jun 2002 & AA- \\
\hline Czech Republic & 26 ago 2005 & A & Latvia & 17 ago 2007 & BBB+ \\
\hline Denmark & 10 nov 2003 & AAA & Lebanon & 14 jul 2006 & B- \\
\hline Dominican Republic & 26 set 2006 & B & Lesotho & 18 set 2006 & BB- \\
\hline Ecuador & 30 out 2007 & $\mathrm{CCC}$ & Lithuania & $7 \mathrm{dez} 2007$ & A \\
\hline Egypt & 18 jun 2007 & $\mathrm{BB}+$ & Luxembourg & 21 set 2000 & AAA \\
\hline El Salvador & 31 jan 2005 & $\mathrm{BB}+$ & Macedonia & 14 ago 2007 & $\mathrm{BB}+$ \\
\hline Estonia & 31 ago 2006 & $\mathrm{~A}$ & Malawi & 6 mar 2007 & $\mathrm{~B}-$ \\
\hline
\end{tabular}

Revista Economia Ensaios, Uberlândia (MG), 30 (1), p. 66-94, Jul./Dez. 2015 
Quadro 3 (cont.): Rating da Dívida Soberana no final de 2007

\begin{tabular}{|c|c|c|c|c|c|}
\hline País & Data da atribuição do Rating & Rating & País & Data da atribuição do Rating & Rating \\
\hline Malaysia & 8 nov 2004 & A- & Sri Lanka & 27 abr 2006 & BB- \\
\hline Mali & 30 abr 2004 & B- & Suriname & 18 jun 2004 & B \\
\hline Malta & 12 jul 2007 & A+ & Sweden & 8 mar 2004 & AAA \\
\hline Mexico & 19 set 2007 & BBB+ & Switzerland & 21 set 2000 & AAA \\
\hline Moldova & 18 jun 2007 & B- & Taiwan & 19 nov 2001 & A+ \\
\hline Mongolia & 20 set 2007 & $\mathrm{~B}+$ & Thailand & 22 out 2006 & BBB+ \\
\hline Morocco & 19 abr 2007 & BBB- & Tunisia & 24 mai 2001 & BBB \\
\hline Mozambique & 15 jul 2003 & B & Turkey & $12 \operatorname{dez} 2007$ & BB- \\
\hline Namibia & $7 \mathrm{dez} 2005$ & BBB- & Uganda & 17 mar 2005 & B \\
\hline Netherlands & 21 set 2000 & AAA & Ukraine & 25 out 2006 & BB- \\
\hline New Zealand & 18 ago 2003 & $\mathrm{AA}+$ & United Kingdom & 21 set 2000 & AAA \\
\hline Nigeria & 30 jan 2006 & BB- & United States & 21 set 2000 & AAA \\
\hline Norway & 21 set 2000 & AAA & Uruguay & 27 jul 2007 & BB- \\
\hline Panama & $3 \operatorname{dez} 2003$ & $\mathrm{BB}+$ & Venezuela & 18 out 2007 & BB- \\
\hline Papua New Guinea & 9 jan 2007 & B & Vietnam & 6 nov 2003 & BB- \\
\hline Peru & 6 mar 2007 & $\mathrm{BB}+$ & & & \\
\hline Philippines & 13 fev 2006 & BB & & & \\
\hline Poland & 18 jan 2007 & A- & & & \\
\hline Portugal & 1 mai 2007 & AA & & & \\
\hline Romania & 31 ago 2006 & BBB & & & \\
\hline Russia & 25 jul 2006 & BBB+ & & & \\
\hline Rwanda & $16 \mathrm{dez} 2006$ & B- & & & \\
\hline San Marino & 10 jan 2001 & AA & & & \\
\hline Saudi Arabia & 31 jul 2007 & A+ & & & \\
\hline Serbia & 19 mai 2005 & BB- & & & \\
\hline Singapore & 14 mai 2003 & AAA & & & \\
\hline Slovakia & 23 jul 2007 & A & & & \\
\hline Slovenia & 12 jul 2006 & AA & & & \\
\hline South Africa & 25 jul 2007 & BBB+ & & & \\
\hline Spain & $10 \mathrm{dez} 2003$ & AAA & & & \\
\hline
\end{tabular}

Fonte: www.fitchratings.com; Elaboração do autor 
Quadro 4: Relação das Taxas de Juros Nominais

\begin{tabular}{|c|c|c|}
\hline País & Taxa de Juros Utilizada & Código da Série Utilizada \\
\hline Afghanistan, I.R. of & MONEY MARKET RATE & 51260B..ZF $\ldots$ \\
\hline Algeria & MONEY MARKET RATE & 61260B..ZF... \\
\hline Anguilla & MONEY MARKET RATE & 31260B..ZF... \\
\hline Antigua and Barbuda & MONEY MARKET RATE & 31160B..ZF... \\
\hline Argentina & MONEY MARKET RATE & 21360B..ZF... \\
\hline Armenia & MONEY MARKET RATE & 91160B..ZF... \\
\hline Aruba & MONEY MARKET RATE & 31460B..ZF... \\
\hline Australia & AVERAGE RATE ON MONEY MARKET & 19360B..ZF... \\
\hline Austria & MONEY MARKET RATE & 12260B..ZF... \\
\hline Bahrain, Kingdom of & MONEY MARKET (INTERBANK) RATE & 41960B..ZF... \\
\hline Belgium & CALL MONEY RATE & 12460B..ZF... \\
\hline Benin & OVERNIGHT ADVANCES & 63860B..ZF... \\
\hline Bolivia & MONEY MARKET RATE & 21860B..ZF... \\
\hline Brazil & MONEY MARKET RATE & 22360B..ZF... \\
\hline Bulgaria & INTERBANK RATE & 91860B..ZF... \\
\hline Burkina Faso & OVERNIGHT ADVANCES & 74860B..ZF... \\
\hline Canada & OVERNIGHT MONEY MARKET RATE & 15660B..ZF... \\
\hline Chile & MONEY MARKET RATE & 22860B..ZF... \\
\hline China,P.R.:Hong Kong & MONEY MARKET RATE & 53260B..ZF... \\
\hline China,P.R.:Macao & INTERBANK RATE (END OF PERIOD) & 54660B..ZF... \\
\hline Colombia & INTERBANCARIA (TBS) & 23360B..ZF... \\
\hline Congo, Dem. Rep. of & INTERBANK MARKET RATE - MAXIMUM & 63660BB.ZF... \\
\hline Côte d'Ivoire & OVERNIGHT ADVANCES & 66260B..ZF... \\
\hline Croatia & MONEY MARKET RATE & $96060 B . . Z F \ldots$ \\
\hline Cyprus & MONEY MARKET RATE & 42360B..ZF... \\
\hline Czech Republic & MONEY MARKET RATE & 93560B..ZF... \\
\hline Denmark & CALL MONEY RATE & 12860B..ZF ... \\
\hline Dominica & MONEY MARKET RATE & 32160B..ZF... \\
\hline Dominican Republic & MONEY MARKET RATE & 24360B..ZF... \\
\hline ECCU & INTERBANK RATE & 30960B..ZF... \\
\hline El Salvador & MONEY MARKET RATE & 25360B..ZF... \\
\hline Estonia & MONEY MARKET RATE & 93960B..ZF... \\
\hline Euro Area & INTERBANK RATE (3-MONTH MATURITY) & 16360B..ZF... \\
\hline Fiji & OVERNIGHT INTERBANK RATE & 81960B..ZF... \\
\hline Finland & AVERAGE COST OF CB DEBT & 17260B..ZF... \\
\hline France & CALL MONEY RATE & 13260B..ZF... \\
\hline Georgia & MONEY MARKET RATE & 91560B..ZF... \\
\hline Germany & CALL MONEY RATE & 13460B..ZF... \\
\hline Ghana & MONEY MARKET RATE & 65260B..ZF... \\
\hline Greece & MONEY MARKET RATE (3 MTH INTERBANK) & 17460B..ZF... \\
\hline Grenada & MONEY MARKET RATE & 32860B..ZF... \\
\hline Guatemala & MONEY MARKET RATE & 25860B..ZF... \\
\hline Guinea-Bissau & MONEY MARKET RATE & 65460B..ZF... \\
\hline Iceland & MONEY MARKET RATE & 17660B..ZF... \\
\hline India & CALL MONEY RATE & 53460B..ZF... \\
\hline Indonesia & CALL MONEY RATE & 53660B..ZF... \\
\hline Ireland & 1 MONTH FIXED RATE & 17860B..ZF ... \\
\hline Italy & MONEY MARKET RATE & 13660B..ZF... \\
\hline Jamaica & MONEY MARKET RATE & 34360B..ZF... \\
\hline Japan & CALL MONEY RATE & 15860B..ZF... \\
\hline Jordan & MONEY MARKET RATE & 43960B..ZF... \\
\hline Korea, Republic of & MONEY MARKET RATE & 54260B..ZF... \\
\hline Kuwait & INTERBANK DEPOSIT RATE (3 M) & 44360B..ZF... \\
\hline Kyrgyz Republic & MONEY MARKET RATE & 91760B..ZF... \\
\hline Latvia & MONEY MARKET RATE & $94160 \mathrm{~B} . . Z \mathrm{ZF} . .$. \\
\hline Libya & INTERBANK CALL LOANS:MAXRATE & 67260B..ZF... \\
\hline Lithuania & MONEY MARKET RATE & 94660B..ZF... \\
\hline Luxembourg & INTERBANK RATE & 13760B..ZF... \\
\hline
\end{tabular}


Quadro 4 (cont.): Relação das Taxas de Juros Nominais

\begin{tabular}{|c|c|c|}
\hline País & Taxa de Juros Utilizada & Código da Série Utilizada \\
\hline Madagascar & MONEY MARKET RATE & 67460B..ZF... \\
\hline Malaysia & INTERBANK OVERNIGHT MONEY & 54860B..ZF... \\
\hline Maldives & CALL MONEY RATE & 55660B..ZF... \\
\hline Mali & OVERNIGHT ADVANCES & 67860B..ZF... \\
\hline Mauritius & MONEY MARKET RATE & 68460B..ZF... \\
\hline Mexico & BANKERS' ACCEPTANCES & 27360B..ZF... \\
\hline Moldova & MONEY MARKET RATE & 92160B..ZF... \\
\hline Montserrat & MONEY MARKET RATE & 35160B..ZF... \\
\hline Morocco & MONEY MARKET RATES & 68660B..ZF... \\
\hline Mozambique & MONEY MARKET RATE & 68860B..ZF... \\
\hline Namibia & MONEY MARKET RATE & 72860B..ZF... \\
\hline Netherlands & CALL MONEY RATE & 13860B..ZF... \\
\hline New Zealand & MONEY MARKET RATE & 19660B..ZF... \\
\hline Niger & OVERNIGHT ADVANCES & 69260B..ZF... \\
\hline Norway & CALL MONEY RATE & 14260B..ZF... \\
\hline Oman & OVERNIGHT INTERBANK LENDING RATE NC & 44960B..ZF... \\
\hline Pakistan & CALL MONEY RATE & 56460B..ZF... \\
\hline Panama & MONEY MARKET RATE & 28360B..ZF... \\
\hline Papua New Guinea & INTERBANK RATE AVERAGE & 85360B..ZF... \\
\hline Paraguay & MONET MARKET RATE & 28860B..ZF... \\
\hline Peru & INTERBANK RATE NC & 29360B..ZF... \\
\hline Philippines & MONEY MARKET RATE & 56660B..ZF... \\
\hline Poland & MONEY MARKET RATE & 96460B..ZF... \\
\hline Portugal & UP TO 5 DAYS INTERBANK DEPOSIT & 18260B..ZF... \\
\hline Qatar & MONEY MARKET RATE & 45360B..ZF... \\
\hline Romania & MONEY MARKET RATE & 96860B..ZF... \\
\hline Russian Federation & MONEY MARKET RATE & 92260B..ZF... \\
\hline Rwanda & INTERBANK MARKET & 71460B..ZF... \\
\hline Senegal & MONEY MARKET RATE & 72260B..ZF... \\
\hline Serbia, Republic of & MONEY MARKET RATE & 94260B..ZF... \\
\hline Singapore & 3 MONTH INTERBANK RATE & 57660B..ZF... \\
\hline Slovak Republic & MONEY MARKET RATE & 93660B..ZF... \\
\hline Slovenia & MONEY MARKET RATE & 96160B..ZF... \\
\hline South Africa & MONEY MARKET RATE & 19960B..ZF... \\
\hline Spain & CALL MONEY RATE & 18460B..ZF... \\
\hline Sri Lanka & INTERBANK CALL LOANS & 52460B..ZF... \\
\hline St. Kitts and Nevis & MONEY MARKET RATE & 36160B..ZF... \\
\hline St. Lucia & MONEY MARKET RATE & 36260B..ZF... \\
\hline St. Vincent \& Grens. & MONEY MARKET RATE & 36460B..ZF... \\
\hline Suriname & MONEY MARKET RATE & 36660B..ZF... \\
\hline Swaziland & MONEY MARKET RATE & 73460B..ZF... \\
\hline Sweden & CALL MONEY RATE & 14460B..ZF... \\
\hline Switzerland & MONEY MARKET RATE & 14660B..ZF... \\
\hline Thailand & MONEY MARKET RATE & 57860B..ZF... \\
\hline Togo & OVERNIGHT ADVANCES & 74260B..ZF... \\
\hline Tunisia & MONEY MARKET RATE & 74460B..ZF... \\
\hline Turkey & INTERBANK MONEY MARKET RATE & 18660B..ZF... \\
\hline Ukraine & MONEY MARKET RATE & 92660B..ZF... \\
\hline United Kingdom & OVERNIGHT INTERBANK MIN & 11260B..ZF... \\
\hline United States & FEDERAL FUNDS RATE & 11160B..ZF... \\
\hline Uruguay & MONEY MARKET RATE & 29860B..ZF... \\
\hline Vanuatu & INTER-BANK BORROWING RATE(REPRESENT) & 84660B..ZF... \\
\hline Venezuela, Rep. Bol. & MONEY MARKET RATE & 29960B..ZF... \\
\hline WAEMU & MONEY MARKET RATE & 75960B..ZF... \\
\hline Zimbabwe & INTERBANK RATE (3 MONTHS) & 69860B..ZF... \\
\hline
\end{tabular}

Fonte: Elaboração do autor com base nos dados do FMI. 